\title{
AXIOMATIC AND COORDINATE GEOMETRY
}

\author{
KAPIL PARANJAPE
}

\section{INTRODUCTION}

At some point between high school and college we first make the transition between Euclidean (or synthetic) geometry and co-ordinate (or analytic) geometry. Later, during graduate studies we are introduced to differential geometry of many dimensions. The justification given in the first instance is that coordinates are a natural outcome of the axioms of Euclidean geometry; and in the second case because Riemannian geometry is much more general than axiomatic non-Euclidean geometry. In this expository account we examine these two justifications.

First of all due to the existence of non-Euclidean geometries one should take as the starting point not the usual axioms of Euclidean geometry but the "local" axioms proposed by Veblen [5], Hilbert [4] and others. These are satisfied by a small convex region within any of the axiomatic geometriesEuclidean or not. We will then follow (in Section 2) the exposition of Coxeter (see [2]) to show that any such geometry can be embedded within Euclidean space (of dimension 3). Thus there is a choice of coordinates for any such geometry - a justification for the first step above.

Now it is clear that there are many possible choices of coordinates. However, from the various physical and other applications it becomes clear that we must restrict our attention to those changes of coordinates which are differentiable with respect to one another. The search is then on for "differential covariants" or quantities that change in some systematic fashion with such a change of coordinates. An important such invariant is the Riemannian curvature and its other face, the sectional curvature (see Section 3).

We now examine the geometry constructed by axiomatic means to see what the sectional curvatures of this geometry can be. A combination of certain results of Schur, Cartan and Hadamard shows us that indeed we only obtain the "classical" geometries-flat Euclidean space, hyperbolic space (of Lobachevsky and Bolyai) and elliptic space (of Poncelet and Riemann) - the geometries of constant sectional curvature. Thus Riemannian geometry does lead to more general non-Euclidean geometries than those that can be constructed by axiomatic means. This gives a justification for the second step away from synthetic geometry.

In a final summarising section we suggest some additional reading and also point out some other interesting ways of constructing geometries that have not been covered here.

\section{The AXiomatic APPROACH}

In order to avoid the many choices for the axiom of parallels we look at those axioms that are satisfied by a convex region of space (see Figure 1) in any axiomatic geometry-Euclidean or not. The basic undefined entities are points, lines and planes and the undefined relations among them are incidence (to-lie-on) and separation (between-ness). The entities and relations are made clear by the following axioms. 


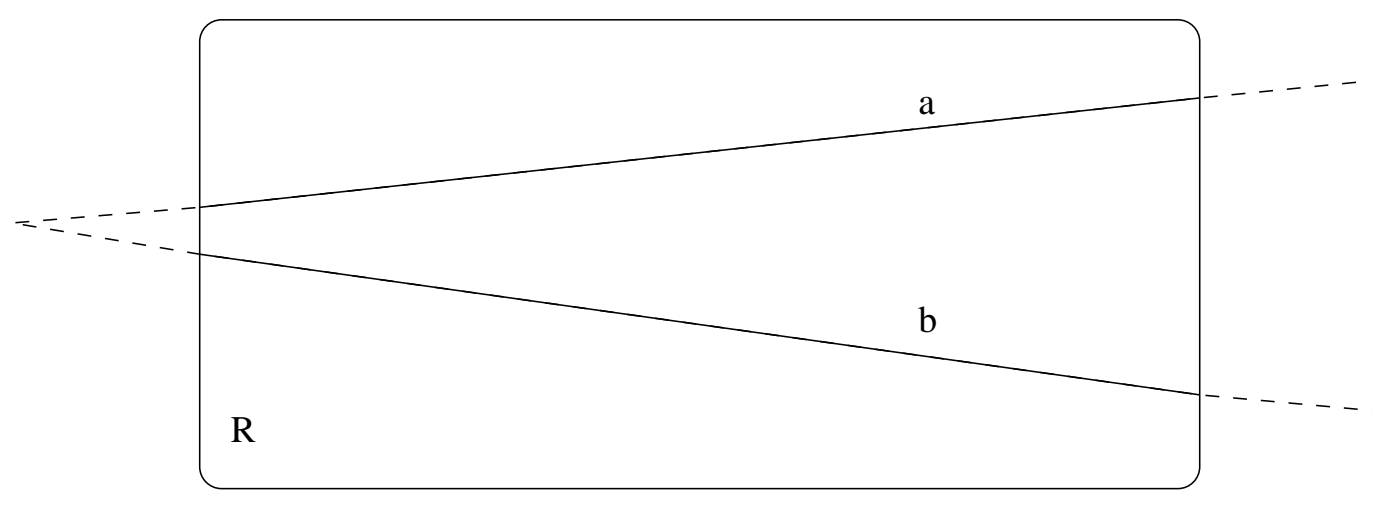

\section{$\mathrm{R}$ : a convex region $\quad \mathrm{a} \& \mathrm{~b}$ : lines which do not meet in $\mathrm{R}$}

FiguRE 1. The geometry of a convex region

We begin with the axioms of incidence:

(1) Any two points lie on exactly one line and any line has at least two points on it.

(2) Any three points not all on one line lie on exactly one plane and any plane has at least three non-collinear points on it.

(3) If two points of a line are on a plane then every point of the line is on the plane (we then say that the line is on the plane).

Then we have the axioms of dimension:

(1) There are at least four points which are non-collinear and non-coplanar.

(2) If two planes meet then they meet in at least a pair of points.

The axioms of order and separation:

(1) If a point $B$ is between the points $A$ and $C$ then $A, B, C$ are collinear and $B$ is between $C$ and $A$.

(2) If $A, B, C$ are distinct collinear points then exactly one is between the other two.

(3) If $A$ and $B$ are distinct points there is at least one point $C$ so that $B$ is between $A$ and $C$ and a point $D$ so that $D$ is between $A$ and $B$.

(4) If $A, B, C$ are non-collinear points and $l$ is a line in the plane of $A, B, C$ so that none of these points lie on $l$, then if $l$ contains a point between $A$ and $B$ then it must contain a point between $A$ and $C$ or a point between $B$ and $C$.

Of these the last axiom is rather special and is called Pasch's axiom (see Figure 2). We shall see later that this is the one property characterising planes in axiomatic geometry which shows that there are no planes in general Riemannian geometry.

Finally, we have the Archimedean least upper bound principle which gives us the least upper bound property for points on a line:

Given a sequence of points $A_{n}$ and a point $B$ such that $A_{n+1}$ is between $B$ and $A_{n}$ for all $n$, there is a point $C$ such that $C$ is between $B$ and $A_{n}$ for all $n$ and for all points $D$ lying between $B$ and $A_{n}$ for all $n, D$ is between $B$ and $C$. 


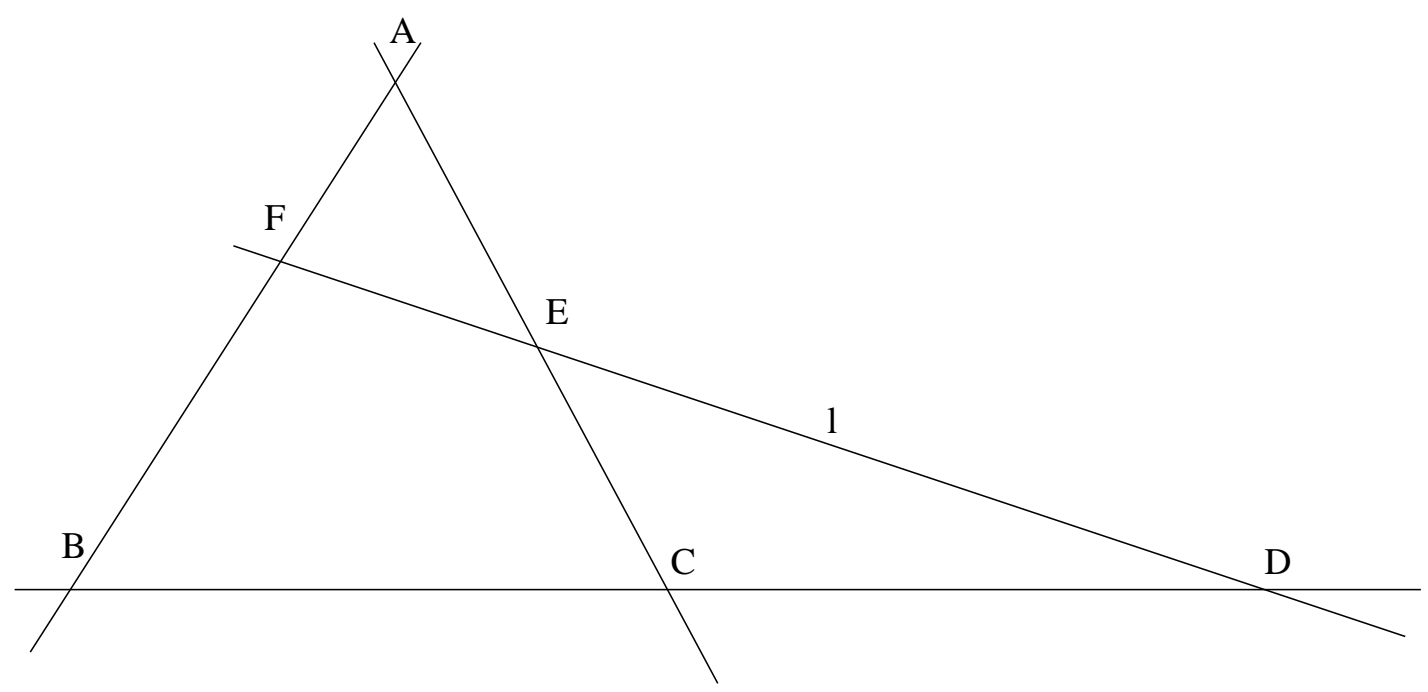

Figure 2. Pasch's axiom of separation

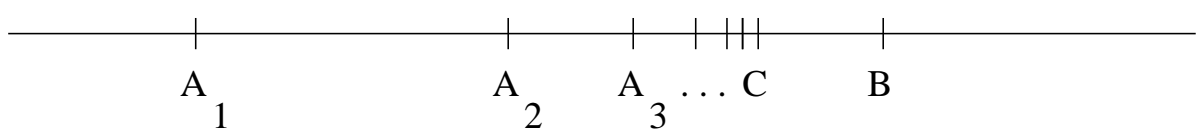

Figure 3. Archimedean least upper bound principle

A much briefer list of equivalent axioms was provided by Veblen using only the notion of points and the relation of between-ness; we use the symbol $[A B C]$ to denote $B$ lies between $A$ and $C$.

(1) There are at least two distinct points.

(2) For any two distinct points $A$ and $B$ there is a point $C$ so that $[A B C]$.

(3) If $[A B C]$ then $A, B$ and $C$ are distinct.

(4) If $[A B C]$ then $[C B A]$ but not $[B C A]$.

Defn. We say that $C$ lies on the line $l(A B)$ if $C=A$ or $C=B$ or $[A B C]$ or $[A C B]$ or $[C A B]$. A pair of lines is said to meet if they have a point in common. If $A, B, C$ all lie on a line we say that they are collinear.

(5) If $C$ and $D$ are distinct points on the line $l(A, B)$ then $A$ lies on $l(C, D)$.

(6) There is at least one point not on $l(A, B)$.

(7) If $A, B, C$ are non-collinear and $D, E$ are points so that $[B C D]$ and $[A E C]$ then there is a point $F$ so that $[A F B]$ and $F$ lies on the line $l(D, E)$.

Defn. If $A, B, C$ are non-collinear points then a point $D$ is said to be coplanar with $A, B, C$ if it lies on a line which meets two out of the three lines $l(A, B), l(B, C), l(A, C)$. The locus of all such points is called the plane determined by the three non-collinear points $A, B, C$.

(8) There is at least one point not on the plane determined by three non-collinear points $A, B$, $C$.

(9) Any two planes which meet have at least two distinct points in common. 
(10) Let the points of a line be divided into two disjoint classes each of which satisfy: if $A$ and $B$ lie in the class the so does every point $C$ such that $[A C B]$. Then there is a point $O$ on the line and a pair of points $P$ and $N$ such that $[N O P]$ and the two classes consists of all points between $N$ (respectively $P$ ) and $O$. (In addition $O$ lies in one of the classes.)

To check that a geometry conforms to these axioms is obviously easier than to check the same for Hilbert's axioms; but the equivalence of the two systems shows us that the former is enough.

Now we will outline how the points, lines and planes of such a geometry can be realised as the points, lines and planes of a convex region in coordinate 3 -space preserving all the relations of incidence and between-ness; that is to say we have an embedding of our geometry into that of coordinate 3-space.

The first step is to construct a geometry consisting of "ideal" points, lines and planes; these would have "existed" if our geometry were not confined to a region.

The collection of all lines passing through a fixed point has the following properties:

(1) Any pair of lines in this collection are coplanar.

(2) For every point there is a line from this collection that contains it.

Thus we define an "ideal point" or Point to be a collection of lines with the above two properties (see Figure 4$)$. Any pair $\left(L_{1}, L_{2}\right)$ of distinct coplanar lines then determines a Point constructed as follows:

(1) We take any plane $p_{1}$ containing $L_{1}$ and a different plane $p_{2}$ containing $L_{2}$ which meets $p_{1}$. Then we add the line of intersection $L_{3}=p_{1} \cap p_{2}$ to our collection.

(2) Let $p$ be the plane containing $L_{1}$ and $L_{2}$ and $p^{\prime}$ be any other plane containing a line $L_{3}$ constructed as above, such that $p^{\prime}$ meets $p$. We add the line of intersection $L_{4}=p \cap p^{\prime}$ to out collection.

We leave it to the reader to check that such a collection indeed satisfies the two properties mentioned above. Moreover, any point clearly gives us a Point as the collection of all lines containing this point.

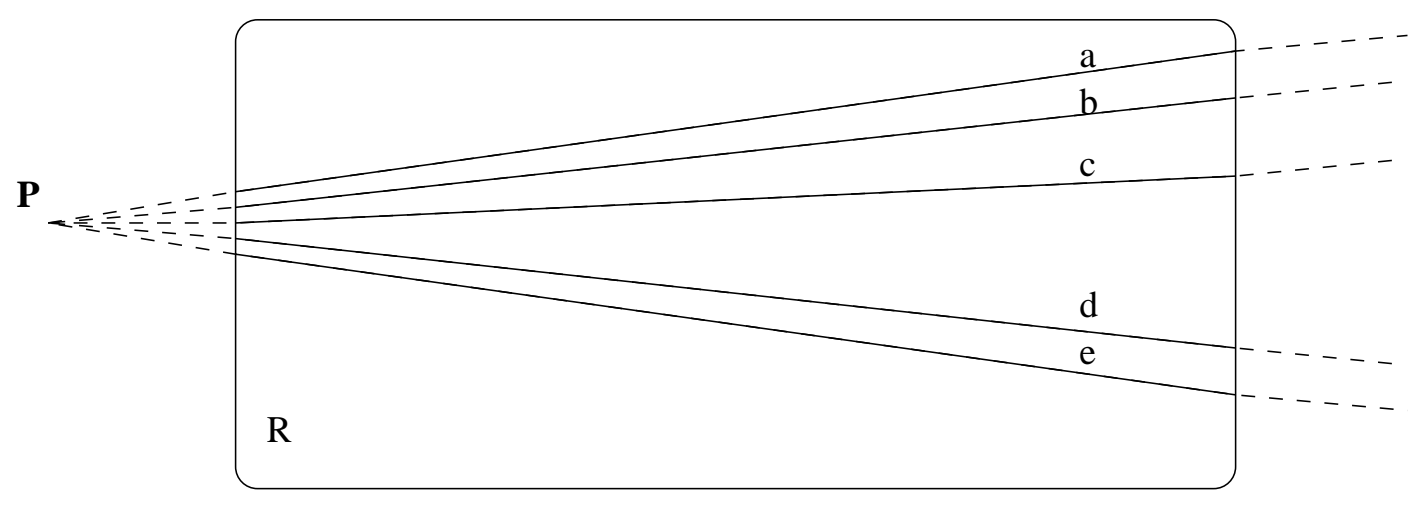

\section{$\mathrm{R}$ : a convex region $\quad$ a,b,c,d,e: lines in $\mathrm{R} \quad \mathbf{P}$ :a Point where a,b,c,d,e meet}

Figure 4. A collection of lines determines a Point

Given a pair of distinct Points A and $\mathbf{B}$ we consider those planes which contain a line each from the collections corresponding to A and B. The collection of all these planes determine an "ideal line" or Line. Any pair $\left(p_{1}, p_{2}\right)$ of distinct planes then determines a Line constructed as follows: 
(1) We choose a pair of planes $\left(q_{1}, q_{2}\right)$ such that each $q_{i}$ meets each $p_{j}$.

(2) Let $A_{i}$ be the Point determined by the pair of lines $\left(q_{i} \cap p_{1}, q_{i} \cap p_{2}\right)$.

(3) A plane $p$ belongs to our collection if it contains a line in each of the collections $A_{1}$ and $A_{2}$. We leave it to the reader to check that this construction is independent of the choice of the pair $\left(q_{1}, q_{2}\right)$. Moreover, we also note that the collection of all planes containing a line also satisfies the required conditions and thus determines a Line.

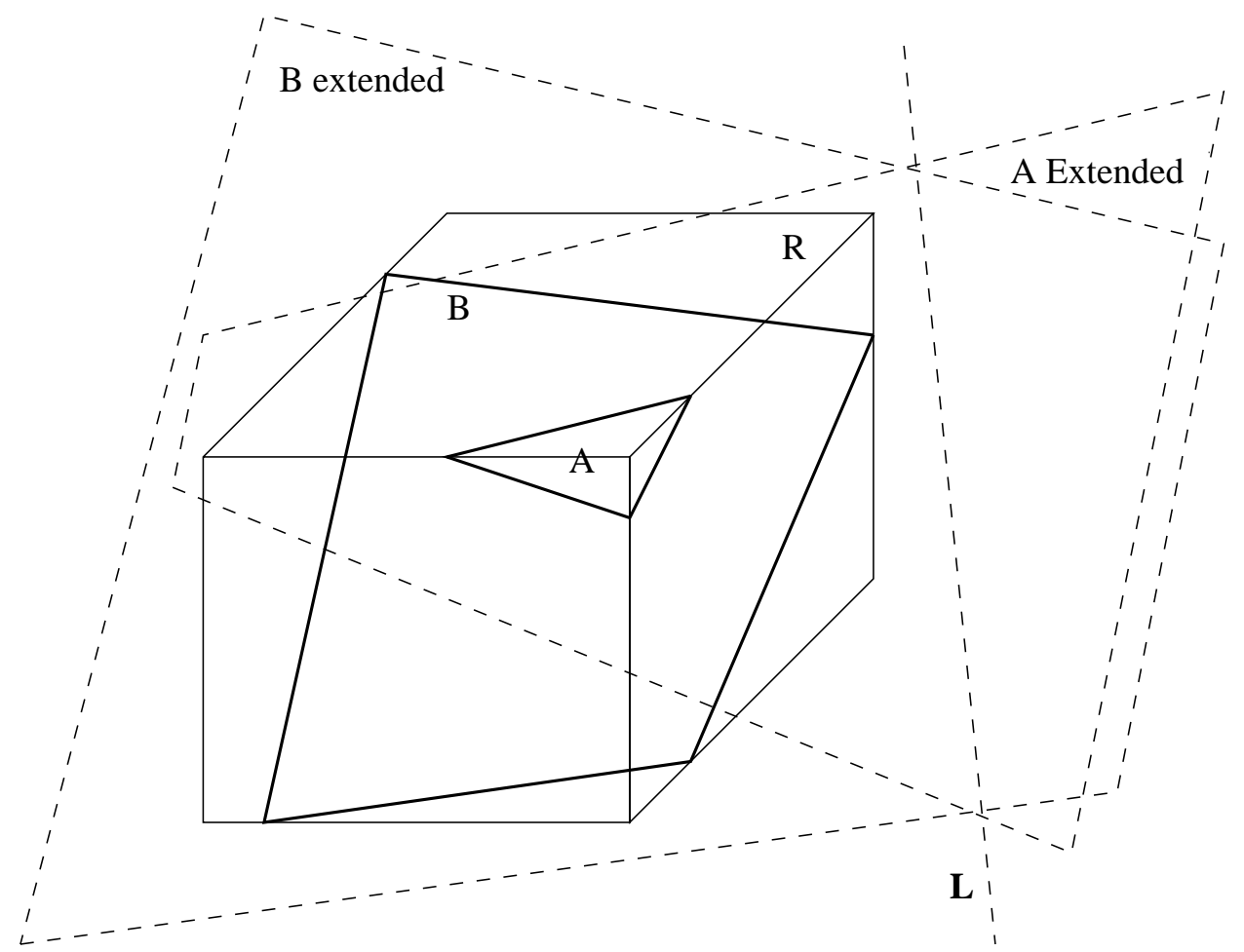

\section{R: a convex region A \& B: planes in $\mathrm{R} \quad \mathbf{L}$ : a Line in which A \& B meet}

Figure 5. A collection of planes determines a Line

We say that a Point lies on a Line if every plane that belongs to the collection determining the Line contains a line that belongs to the collection determining the Point. This specifies the notion of incidence for Points and Lines. It is clear that if a Line $\mathbf{L}$ is determined by the line $L^{\prime}$ then $\mathbf{L}$ contains the Point $\mathbf{A}$ if and only if $L^{\prime}$ lies in the collection corresponding to the Point $\mathbf{A}$. In particular, if the Point $\mathbf{A}$ is that determined $A^{\prime}$, then $\mathbf{A}$ lies on $\mathbf{L}$ if and only if $A^{\prime}$ lies on $L^{\prime}$.

Thus we have embedded our given geometry into an "ideal" geometry of Points and Lines with relations of incidence and separation. What are the axioms satisfied by this "ideal" geometry? We claim that the following axioms are satisfied:

(1) Any pair of Points A and $\mathbf{B}$ lie on exactly one Line. We denote this line by $\mathbf{A B}$. 


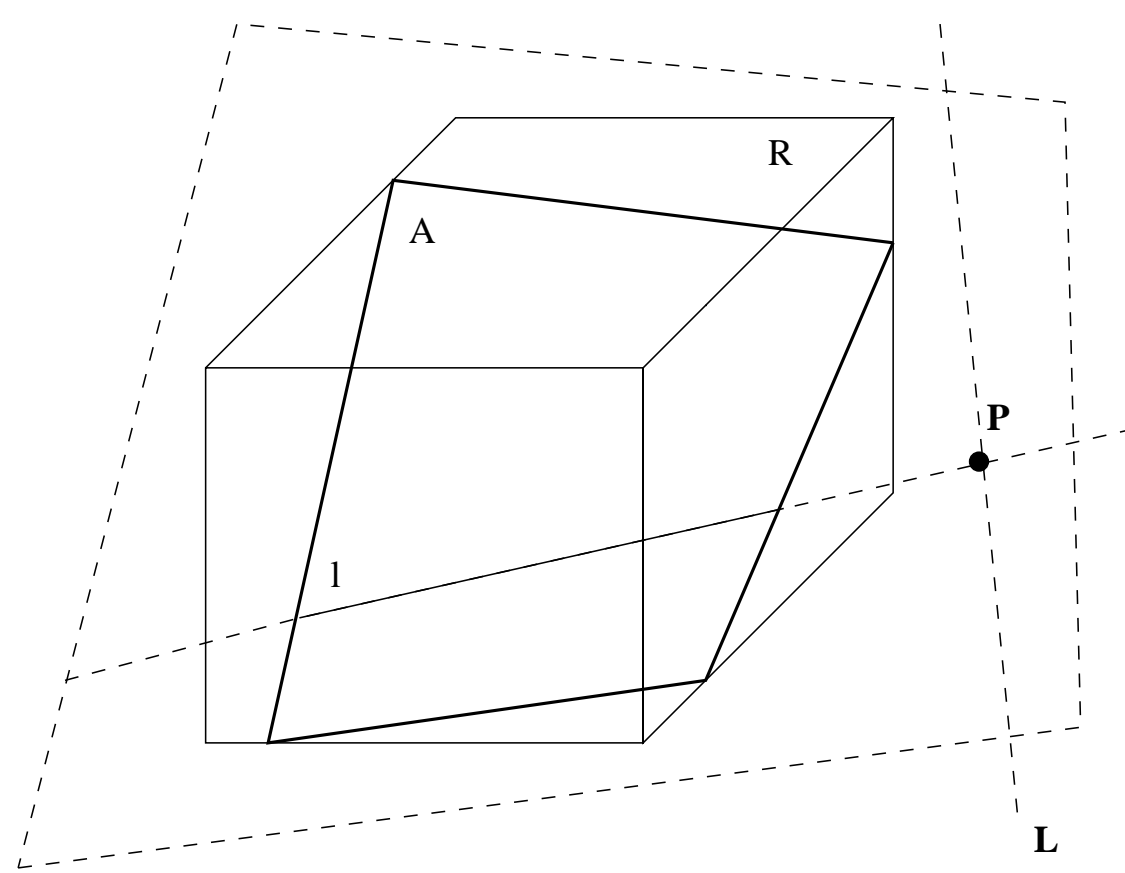

$\mathrm{R}$ : a convex region $\mathrm{A}$ : a plane in $\mathrm{R}$ 1: a line in $\mathrm{R}$

\section{$\mathbf{L}$ : a Line contained in A $\mathbf{P}$ : a Point contained in 1}

\section{1 lies on A and hence $\mathbf{P}$ lies on $\mathbf{L}$}

Figure 6. A Line contains a Point

(2) Given four Points $\mathbf{A}, \mathbf{B}, \mathbf{C}$ and $\mathbf{D}$, the Line $\mathbf{A B}$ meets the Line $\mathbf{C D}$ if and only if the Line AD meets the Line BC. In this case we call the four Points coplanar.

(3) There are five Points so that no four of these are coplanar.

(4) Given any five Points $\mathbf{A}, \mathbf{B}, \mathbf{C}, \mathbf{D}$ and $\mathbf{E}$, there is a point $\mathbf{F}$ on the Line $\mathbf{A B}$ so that the Points $\mathbf{C}, \mathbf{D}, \mathbf{E}$ and $\mathbf{F}$ are coplanar.

The first three axioms are the axioms of incidence and the latter two are the axioms of dimension for Projective geometry of 3 dimensions. It is a well-known theorem (see for example [2]) that such a geometry is isomorphic to the coordinate Projective geometry over a skew-field (that is multiplication need not be commutative).

The relation of separation is a little more intricate. We will try to define the relation picturised in Figure 7. Given two pairs $(\mathbf{A}, \mathbf{B})$ and $(\mathbf{C}, \mathbf{D})$ of Points lying on a Line $\mathbf{L}$; we say that the pairs separate each other if for some plane $p$ in the collection $\mathbf{L}$ and some point $O$ in the plane $p$, there is a line $L^{\prime}$ in $p$ and four points $A^{\prime}, B^{\prime}, C^{\prime}$ and $D^{\prime}$ on $L^{\prime}$ so that:

(1) The lines $O A^{\prime}, O B^{\prime}, O C^{\prime}$ and $O D^{\prime}$ are in the collections $\mathbf{A}, \mathbf{B}, \mathbf{C}$ and $\mathbf{D}$ respectively.

(2) $B^{\prime}$ and $C^{\prime}$ are between $A^{\prime}$ and $D^{\prime}$, and $B^{\prime}$ is between $A^{\prime}$ and $C^{\prime}$. 
We use the notation $\mathbf{A B} \| \mathbf{C D}$ to denote this relation. Now in case the Points $\mathbf{A}, \mathbf{B}, \mathbf{C}$ and $\mathbf{D}$ correspond to points $A^{\prime}, B^{\prime}, C^{\prime}$ and $D^{\prime}$ respectively, then the two relations of separation coincide; AB $\| \mathbf{C D}$ if and only these points are all collinear, and either $C$ is between $A$ and $B$ while $D$ is not or $D$ is between $A$ and $B$ and $C$ is not.

A C B

$\mathrm{D}$

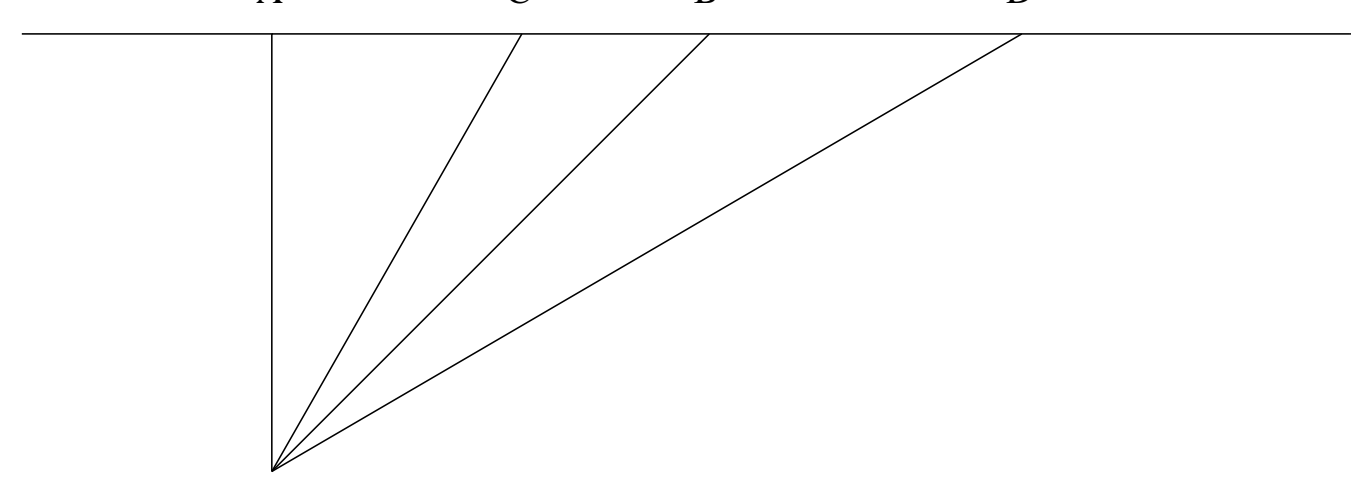

Figure 7. AB $\| \mathbf{C D}$ on a Projective Line

The axioms satisfied by the relation of separation are:

(1) If $\mathbf{A}, \mathbf{B}, \mathbf{C}$ and $\mathbf{D}$ are collinear and distinct then exactly one of the relations $\mathbf{A B} \| \mathbf{C D}$, $\mathbf{A D} \| \mathbf{B C}$ and $\mathbf{A C}|| \mathbf{B D}$ holds.

(2) If $\mathbf{A}, \mathbf{B}$ and $\mathbf{C}$ are collinear then there is a Point $\mathbf{D}$ so that $\mathbf{A B} \| \mathbf{C D}$.

(3) If $\mathbf{A B} \| \mathbf{C D}$ and $\mathbf{O}$ is a Point, and $\mathbf{L}$ any Line so that the Points $\mathbf{A}^{\prime}, \mathbf{B}^{\prime}, \mathbf{C}^{\prime}, \mathbf{D}^{\prime}$ of intersection of $\mathbf{L}$ with the Lines $\mathbf{O A}, \mathbf{O B}, \mathbf{O C}$ and $\mathbf{O D}$ respectively are distinct, then $\mathbf{A}^{\prime} \mathbf{B}^{\prime} \| \mathbf{C}^{\prime} \mathbf{D}^{\prime}$ holds.

(4) Given a sequence of Points $\mathbf{A}_{n}$ and Points $\mathbf{B}$ and $\mathbf{O}$ such that $\mathbf{A}_{n} \mathbf{B} \| \mathbf{A}_{n+1} \mathbf{O}$ holds for all $n$, then there is a Point $\mathbf{C}$ so that $\mathbf{A}_{n} \mathbf{B} \| \mathbf{C O}$ holds for all $n$; and so for all points $\mathbf{D}$ so that $\mathbf{A}_{n} \mathbf{B} \| \mathbf{D O}$ holds for all $n$, we have $\mathbf{C B} \| \mathbf{D O}$

The first three are the axioms of separation for Projective geometry and the last is a form of the Archimedean least upper bound principle. These axioms assure us that the base skew-field of our projective space geometry is a complete ordered skew-field - which is then a (commutative) field. Moreover, under some set theoretic assumptions about cardinality this is just the field of real numbers.

Thus our "ideal" geometry is none other than coordinate Projective geometry of of dimension 3 over the field of real numbers. Hence we have an embedding of the given geometry into real Projective space geometry.

The collection of all Points on a Line thus forms a real Projective line which cannot be totally ordered in a way so as to satisfy the Archimedean least upper bound principle. Thus for any line there is at least one Point $\mathbf{A}$ which lies on the corresponding Line that does not correspond to a point. Moreover, if $C$ and $D$ are points on the line then every Point $\mathbf{B}$ so that $\mathbf{A B} \| \mathbf{C D}$ corresponds to a point $B$. In other words the geometry we have is that of a convex subset of Projective space. For any such subset one can find a Projective plane that does not meet it. Thus the convex subset is actually contained in real Affine space; in other words we have a coordinatisation of our geometry. 


\section{Riemannian Geometry}

Let us now consider a small region $M$ of space on which it is possible to put coordinates, say $\left(x_{1}, x_{2}, x_{3}\right)$. Moreover, any other choice of coordinates, say $\left(y_{1}, y_{2}, y_{3}\right)$ is differentiable (to any order) with respect to the given choice, that is to say the $y_{i}$ are differentiable functions of the $x_{i}$, and vice versa. Then we have the non-singularity of the Jacobian matrix

$$
J(y, x)=\left(\begin{array}{lll}
\frac{\partial y_{1}}{\partial x_{1}} & \frac{\partial y_{1}}{\partial x_{2}} & \frac{\partial y_{1}}{\partial x_{3}} \\
\frac{\partial y_{2}}{\partial x_{1}} & \frac{\partial y_{2}}{\partial x_{2}} & \frac{\partial y_{2}}{\partial x_{3}} \\
\frac{\partial y_{3}}{\partial x_{1}} & \frac{\partial y_{3}}{\partial x_{2}} & \frac{\partial y_{3}}{\partial x_{3}}
\end{array}\right) .
$$

The basic geometric notion other than that of points of the space is the notion of tangent vectors at points. At any point these represent the physical notion of instantaneous velocities and form a linear space of dimension 3 called the tangent space at the given point. In some sense this space is the Euclidean approximation of our space at the point.

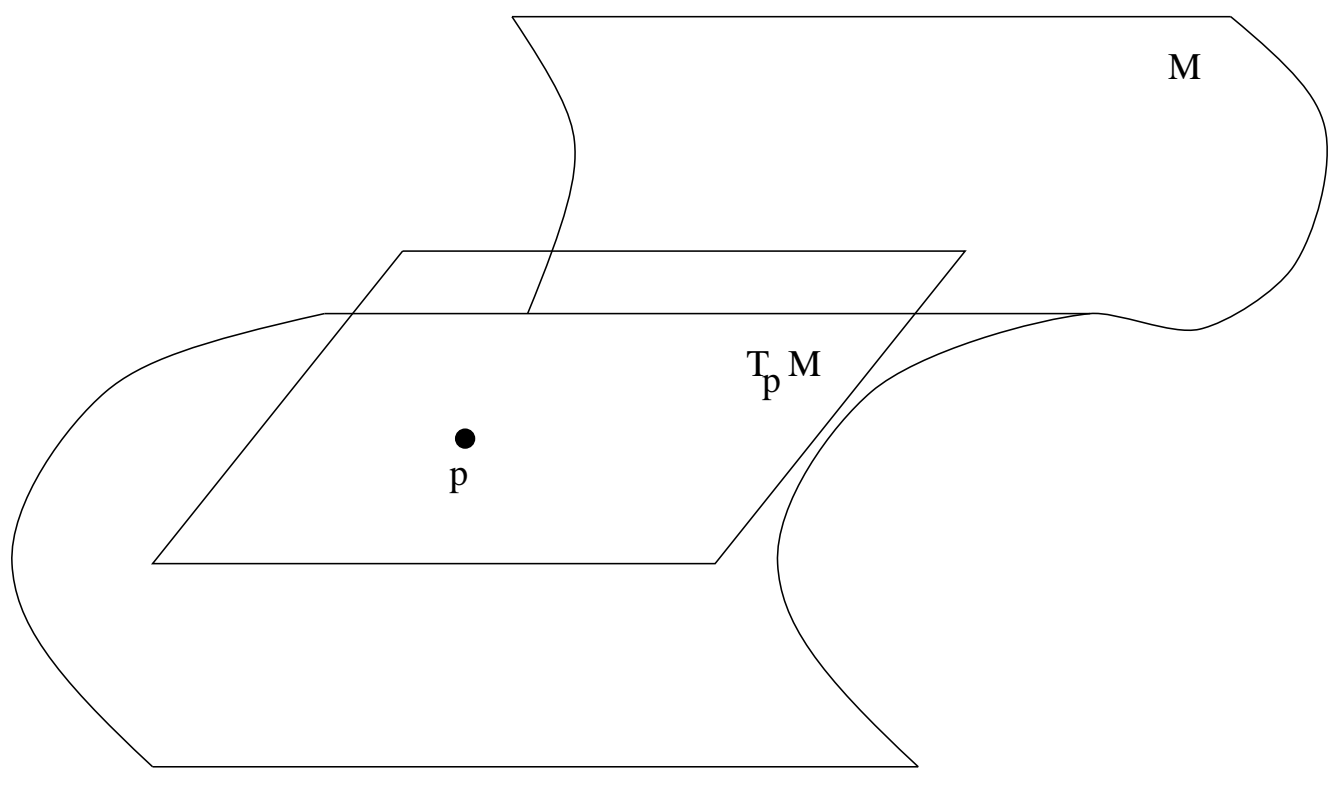

$\mathrm{T}_{\mathrm{p}} \mathrm{M}:$ the tangent space to $\mathrm{M}$ at $\mathrm{p}$

Figure 8. The tangent space to a space

With respect to the given choice of coordinates $\left(x_{1}, x_{2}, x_{3}\right)$ a vector $\vec{v}$ can be represented by a column vector $\mathbf{a}=\left(a_{1}, a_{2}, a_{3}\right)^{t}$. A basis for the tangent vector space at all points is given by the standard basis $\left\{e^{1}, e^{2}, e^{3}\right\}$ of $\mathbf{R}^{3}$. We are also interested in tensor fields, which are sums of terms of the form $f_{i_{1}, i_{2}, \ldots, i_{r}} e^{i_{1}} \otimes e^{i_{2}} \otimes \cdots \otimes e^{i_{r}}$ where $f_{i_{1}, i_{2}, \ldots, i_{r}}$ is a differentiable function. The number $r$ is called the order of the corresponding tensor. A tensor of order 0 is thus just a differentiable function and one of order 1 is a (tangent) vector field. 
In another system of coordinates $\left(y_{1}, y_{2}, y_{3}\right)$ the vector $\vec{v}$ is given by the column vector $J(x, y) \cdot \mathbf{a}$. This can be extended to tensors in an obvious way. With this understanding we now fix a choice of coordinates and a corresponding representation of tangent vectors as column vectors.

In order to measure the magnitude and angle of velocities we are given a symmetric positive definite bilinear pairing $<,>$ on the tangent space at each point. With respect to a given representation of tangent vectors as column vectors, this is given by a positive definite symmetric matrix $\left(g_{i j}\right)_{i, j=1}^{i, j=3}$ of functions on the space. Moreover, we further assume that the matrix entries $g_{i j}$ are differentiable (to any order) functions of the coordinates. Such a pairing is called a Riemannian metric. Having prescribed this we have a (local) Riemannian manifold.

The pairing $<,>$ also extends to pairing on the various types of tensors by induction on the order. In fact we can pair a tensor of order $r$ with a tensor of order $s$ to obtain a tensor of order $|r-s|$.

For any vector field $V$ we have learned in vector calculus to compute the gradient $\nabla_{\vec{v}}(V)$ with respect to a vector $\vec{v}$ at a point $p$. We have also learned about the gradient of a function $f$. These give an example of a derivation; in other words we have the Liebnitz rule

$$
\nabla_{\vec{v}}(f \cdot V)=\nabla_{\vec{v}}(f) \cdot A(p)+f(p) \cdot \nabla_{\vec{v}}(A)
$$

Now we can clearly extend this to all tensors by applying the Liebnitz rule again as follows,

$$
\nabla_{\vec{v}}(A \otimes B)=\nabla_{\vec{v}}(A) \otimes B(p)+A(p) \otimes \nabla_{\vec{v}}(B)
$$

Finally, we also have the linearity relation

$$
\nabla_{a \vec{v}+b \vec{w}}=a \nabla_{\vec{v}}+b \nabla_{\vec{w}}
$$

Now we can also consider other derivations $D$ on tensor fields which satisfy the above rules. It follows easily from the first rule that any two derivations differ by a linear operator. In other words, any derivation $D$ differs from $\nabla$ by a (linear) map which associates to any tangent vector $\vec{v}$ a linear endomorphism $\omega(\vec{v})$ of the space of tangent vectors; we write this as

$$
D_{\vec{v}}=\nabla_{\vec{v}}+\omega(\vec{v})
$$

where $\omega(\vec{v})(f \cdot V)=f(p) \omega(\vec{v})(V)$ is the connection form of Cartan in this special case. By the second rule such a derivation can be extended to tensor fields. Finally, the last rule says that $\omega(\vec{v})$ is linear in $\vec{v}$.

Given a vector field $V$ we can apply the existence theorem for ordinary differential equations to construct a 1-parameter flow $\phi_{t}$ on our space $M$. This satisfies $\phi_{t+s}=\phi_{t} \circ \phi_{s}$ and $\phi_{0}$ fixes all points. Finally, we have $d \phi_{t}(p) / d t=V\left(\phi_{t}(p)\right)$; which is the ordinary differential equation we have solved. For any tensor field $A$, the following limit exists by differentiability of the quantities involved

$$
L_{V}(A)(p)=\lim _{t \rightarrow 0} \frac{A(p)-\phi_{-t} A\left(\phi_{t}(p)\right)}{t}
$$

and it is called the Lie derivative of $A$ with respect to $V$. For a pair of vector fields $V$ and $W$ it is not difficult to show that $L_{V}(f)(p)=\nabla_{V(p)}(f)$ for any differentiable function $f$ and

$$
L_{V}(W)(p)=\nabla_{V(p)}(W)-\nabla_{W(p)}(V)
$$

for any vector field $W$ (and hence $L_{V}(W)=-L_{W}(V)$ ). Note that $L_{V}(W)$ involves the derivatives of $V$ even though the derivative is being taken (in some sense) with respect to $V$. 


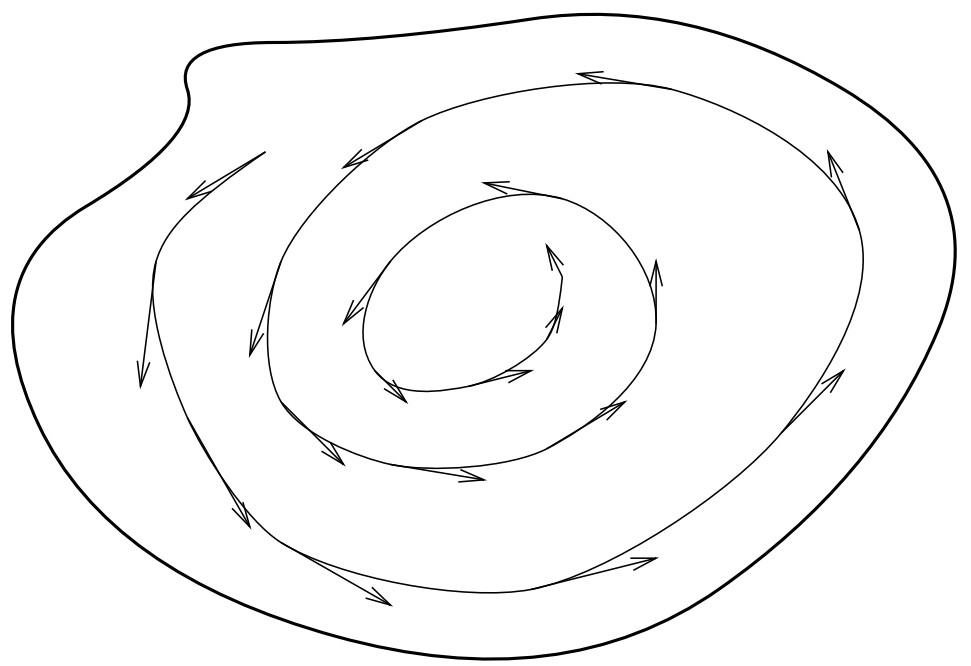

Figure 9. A tangent vector field and its flow

While the gradient of a function is independent of a choice of coordinates because of the interpretation as Lie derivative, the gradient of a tensor of order at least 1 is clearly dependent upon the choice of coordinates, thus it is natural to ask for a derivation $D$ that is in some sense "canonical". This is provided by the condition that the inner product form $<,>$ has no derivative,

$$
D_{\vec{u}}(<V, W>)=<D_{\vec{u}}(V), W>+<V, D_{\vec{u}}(W)>
$$

where $D_{\vec{u}}(f)=\nabla_{\vec{u}}(f)$ as before for any function $f$. In addition we demand the condition that the derivation is torsion-free,

$$
L_{V}(W)(p)=D_{V(p)}(W)-D_{W(p)}(V)
$$

This can be translated into the condition on the connection form $\omega$

$$
\omega(\vec{v})(\vec{w})=\omega(\vec{w})(\vec{v})
$$

There is then a unique such derivation and thus it is independent of any choice of coordinates.

In geometric terms, the usual gradient $\nabla_{\vec{v}}(A)$ measures the deviation of $A(p+t \vec{v})$ from a copy of $A(p)$ moved to the point $p+t \vec{v}$. However, this motion requires a notion of parallel transport or rigid motion - which may be different for our geometry from the Euclidean one provided by the coordinates; the Riemannian metric provides the correct notion of angles and distances and hence of rigid motion. Thus $D$ provides the "corrected" gradient.

Now it is conceivable that there is a choice of coordinates in which the $\omega(\vec{v})$ 's vanish or simplify. Thus we need to find some way of checking this possibility. Riemann introduced the Riemannian curvature as a measure of this. This was modified by Christoffel who defined the curvature operator as follows. First of all for a vector field $V$ and a tensor field $A$ let us define $D_{V}(A)(p)=D_{V(p)}(A)$. We then define the operator $R$ by

$$
R(V, W)(X)=D_{V}\left(D_{W}(X)\right)-D_{W}\left(D_{V}(X)\right)-D_{L_{V}(W)}(X)
$$


One checks that for any functions $f, g$ and $h$ we have

$$
R(f V, g W)(h X)=f g h \cdot R(V, W)(X)
$$

From this it follows that for each point $p, R(V, W)$ defines an endomorphism of the tangent space which depends only on the values $V(p)$ and $W(p)$. We can thus denote this by $R(\vec{v}, \vec{w})$. The Riemannian curvature is then defined by

$$
K(\vec{v}, \vec{w}, \vec{x}, \vec{y})=<R(\vec{v}, \vec{w})(\vec{x}), \vec{y}>
$$

Bianchi proved that $K(\vec{v}, \vec{w}, \vec{x}, \vec{y})=K(\vec{x}, \vec{y}, \vec{v}, \vec{w})$, and for any three vector fields $U, V, W$

$$
\left(D_{U} R\right)(V, W)+\left(D_{W} R\right)(U, V)+\left(D_{V} R\right)(W, U)=0
$$

We now consider the curvature as a function of a pair of tensors of order 2

$$
S(\vec{v} \otimes \vec{w}, \vec{x} \otimes \vec{y})=K(\vec{v}, \vec{w}, \vec{x}, \vec{y})
$$

As usual we introduce the notation

$$
\vec{v} \wedge \vec{w}=\frac{1}{2}(\vec{v} \otimes \vec{w}-\vec{w} \otimes \vec{v})
$$

for skew-symmetric tensors of order 2. Then we have

$$
S(\vec{v} \otimes \vec{w}, \vec{x} \otimes \vec{y})=S(\vec{v} \wedge \vec{w}, \vec{x} \wedge \vec{y})
$$

Moreover $S(\vec{v} \wedge \vec{w}, \vec{x} \wedge \vec{y})$ is a symmetric bilinear pairing on the space of skew-symmetric tensors of order 2. Now one such is given by the pairing of tensors

$$
<\vec{v} \wedge \vec{w}, \vec{x} \wedge \vec{y}>=\frac{1}{2}(<\vec{v}, \vec{x}><\vec{w}, \vec{y}>-<\vec{v}, \vec{y}><\vec{w}, \vec{x}>)
$$

A comparison of the associated quadratic forms is then given by the ratio

$$
\kappa(\vec{v}, \vec{w})=-\frac{K(\vec{v}, \vec{w}, \vec{v}, \vec{w})}{\left\langle\vec{v}, \vec{v}><\vec{w}, \vec{w}>-<\vec{v}, \vec{w}>^{2}\right.}
$$

This is what is called the sectional curvature of the Riemannian manifold (the sign is for reasons of convention). This is by far the most important invariant associated with a Riemannian manifold. It is clear from the above discussion that the sectional curvature, the Riemannian curvature and the Riemann-Christoffel operator on a Riemannian manifold determine each other.

An important result of Cartan and Hadamard (see [3]) states that any Riemannian space $M$ as above with the property that its sectional curvature is constant can be identified with an open subset of either the Euclidean space or Hyperbolic (Bolyai-Lobachevsky) space or Projective space in an isometric way.

We will see in the next section how to apply this to the study of axiomatic geometries.

\section{Manifolds All of Whose GeOdesics ARE LINES}

In order to tie up the first two sections we need to have a notion analogous to lines in a general Riemannian manifold - this is provided by geodesics or energy minimising paths.

To a stationary observer placed on the manifold it would appear that a body travelling along an energy minimising path is subject to no acceleration. The translation of this into differential geometric terms is $D_{X(t)}(X(t))=0$ where $X(t)$ is the tangent vector at time $t$. By the theory of second order 


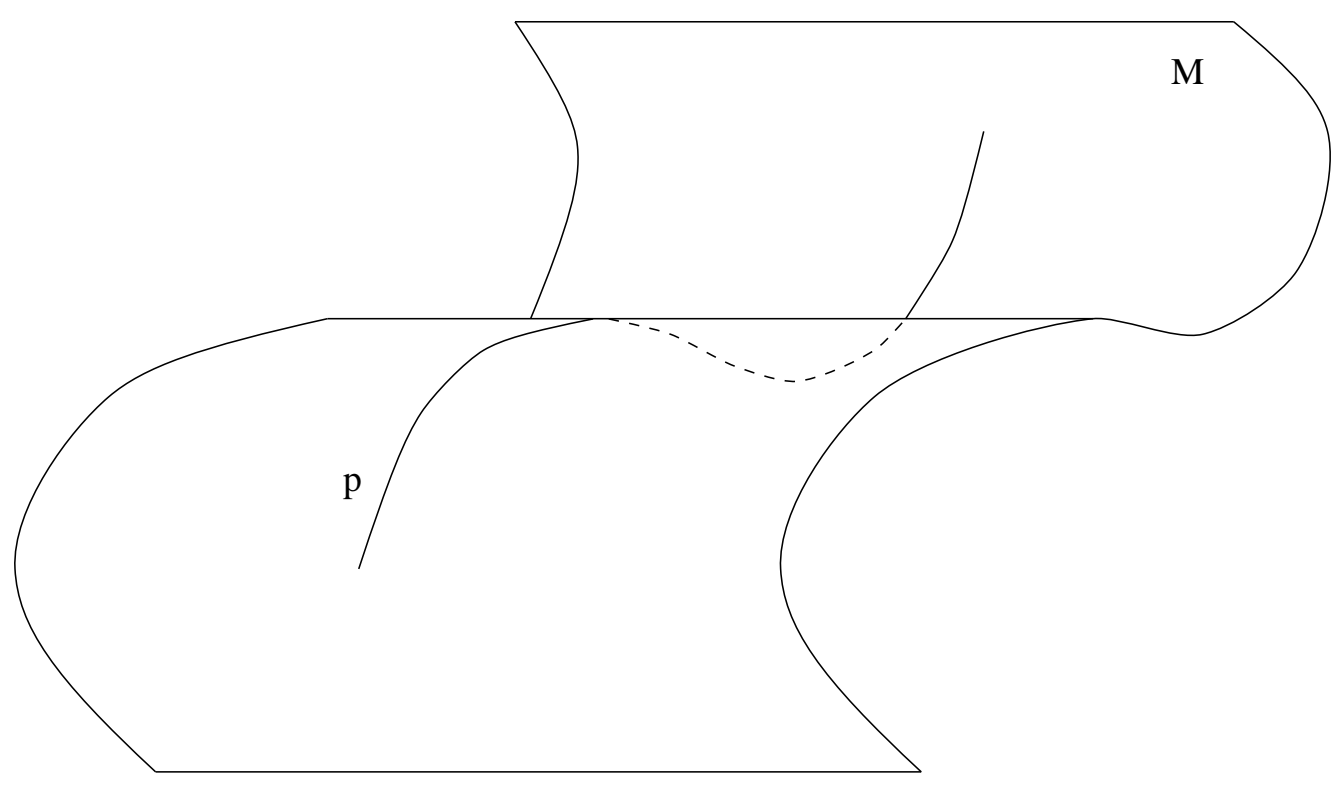

Figure 10. A Geodesic

ordinary differential equations there is a unique geodesic starting at a point $p$ with initial velocity $\vec{v}$ for any choice of $p$ and $\vec{v}$.

A result of Whitehead shows that for any point $p$ there is a small region $M$ surrounding it so that there is a unique geodesic in $M$ joining any pair of points in $M$. The notion of between-ness is defined by saying that $B$ lies between $A$ and $C$ in $M$ if $B$ lies on the (unique) geodesic joining $A$ and $C$. Extending the geodesic within $M$ beyond $B$ and before $A$ gives us the "line" joining $A$ and $B$. We then easily check that Veblen's axioms of local geometry other than those involving planes are satisfied. In particular we have trouble verifying the Pasch axiom - numbered 7 in the list of Veblen's axioms given in section 1 .

Let us therefore make the additional assumption that these axioms dealing with planes are satisfied; we will show that this imposes a restriction on the curvature of the Riemannian manifold $M$, which is satisfied if and only if $M$ is a convex region in one of the "classical" geometries-Euclidean, Hyperbolic or Projective.

According to the results of section 1 we can choose coordinates on $M$ in such a way that the geodesics are mapped into lines. In terms of these coordinates we see that a geodesic must be an accelerated path:

$$
\nabla_{X(t)}(X(t))=D_{X(t)}(X(t))-\omega(X(t))(X(t))=-\omega(X(t))(X(t))
$$

being the acceleration at time $t$. Since the geodesics are lines it follows that acceleration is proportional to $X(t)$; since this is to happen for geodesics in all directions

$$
\omega(\vec{v})(\vec{v})=\lambda_{\vec{v}} \cdot \vec{v}
$$


for some scalar $\lambda_{\vec{v}}$ depending on $\vec{v}$. In addition we have the torsion-free condition and the linearity of $\omega$ in each variable. It follows that $\lambda_{X}=\langle\Lambda, X\rangle$ is a linear functional associated with some vector field $\Lambda$

$$
\omega(\vec{v})(\vec{w})=\frac{1}{2}(<\Lambda(p), \vec{v}>\cdot \vec{w}+<\Lambda(p), \vec{w}>\cdot \vec{v})
$$

Thus we have

$$
\begin{aligned}
R(X, Y) Z=D_{X}\left(D_{Y}(Z)\right)- & D_{Y}\left(D_{X}(Z)\right)-D_{L_{X}(Y)}(Z) \\
= & \frac{1}{2}\left(\left(<D_{X}(\Lambda), Y>-<D_{Y}(\Lambda), X>\right) \cdot Z\right. \\
& +<D_{X}(\Lambda), Z>\cdot Y-<D_{Y}(\Lambda), Z>\cdot X \\
& \quad-<\Lambda, Z>(<\Lambda, X>\cdot Y-<\Lambda, Y>\cdot X))
\end{aligned}
$$

Let $X$ and $Y$ be orthogonal then

$$
K(X, Y, X, Y)=<R(X, Y) X, Y>=<D_{X}(\Lambda), X><Y, Y>-<\Lambda, X>^{2}<Y, Y>
$$

Thus the sectional curvature becomes

$$
\kappa(X, Y)=\frac{<\Lambda, X>^{2}-<D_{X}(\Lambda), X>}{<X, X>^{2}}
$$

where the right hand side does not depend on $Y$ ! Thus by symmetry it does not depend on $X$ either.

Now we have $\kappa(X, Y)(p)=\kappa(p)$ depends only on the point $p$. Thus we have

$$
K(X, Y, Z, W)=\kappa \cdot(<X, W><Y, Z>-<X, Z><Y, W>)
$$

and hence

$$
R(X, Y)(Z)=\kappa \cdot(<Y, Z>X-<X, Z>Y)
$$

Now applying the second identity proved by Bianchi

$$
\begin{gathered}
0=\left(D_{U} R\right)(V, W)(X)+\left(D_{W} R\right)(U, V)(X)+\left(D_{V} R\right)(W, U)(X) \\
=D_{U} \kappa(<V, X>W-<W, X>V)+D_{W} \kappa(<U, X>V-<V, X>U)+ \\
D_{V} \kappa(<W, X>U-<U, X>W)
\end{gathered}
$$

So that if $U=X$ is orthogonal to $V$ and $W$ we get

$$
0=D_{W} \kappa \cdot\left\langle U, U>V-D_{V} \kappa \cdot\langle U, U>W\right.
$$

Finally, since have assumed that we are in (at least) three dimensions we can assume that $V$ and $W$ are linearly independent and $\langle U, U\rangle \neq 0$, so that we obtain $D_{W} \kappa=0$. Since $W$ is arbitrary this just means that $\kappa$ is the constant function. Now, by applying the result of Cartan and Hadamard mentioned in the previous section we see that our local Riemannian space $M$ has to be contained in one of the "classical" geometries - Euclidean, Hyperbolic or Projective. 


\section{Conclusion}

Combining all the results we see that Riemannian geometry is indeed of a more general nature than axiomatic geometry - at least over real numbers and in dimension strictly greater than 2. One can generalise further by dropping the requirement of a Riemannian metric and retaining the linear or affine connection $D$. Yet another generalisation is to consider fields other than tensor fields (for example spinor fields) and consider derivations on such fields.

At the same time a number of interesting questions remain if the field is not that of real numbers (in dimension at least 3) or if we have a planar geometry which does not satisfy Desargues axiom (which is almost obvious in dimension 3) - in the latter case we do not even have a skew-field associated with the geometry. These are the subject of study in Combinatorial Geometry and Group theory. The latter (group theory) points to another approach to geometry - that taken by Pieri, Klein and Liewhere the fundamental notion is that of a group of motions. A similar study to the one undertaken above ought to show that this leads to Lie groups and Symmetric spaces.

Acknowledgements. A. Sitaram encouraged the author to re-examine high school geometry. The use of Schur's result was suggested by M. S. Raghunathan. Discussions with R. R. Simha, T. R. Ramadas and V. Pati were also very helpful.

\section{REFERENCES}

1. E. Artin, Geometric Algebra, Info. Science Publ. Inc., NY, USA, 1957.

2. H. S. M. Coxeter, Non-Euclidean geometry, Univ. of Toronto, Toronto, 1961.

3. N. J. Hicks, Notes on Differential Geometry, Van Nostrand, 1965.

4. D. Hilbert, Foundations of Geometry, Open Court, La Salle, Ill., USA, 1971.

5. O. Veblen and W. Young, Projective Geometry, 2 vols., Ginn and Co., Blaisdell, USA, 1965. 